\title{
Variational method study of the spin-1 Ising model with biaxial crystal-field anisotropy
}

\author{
J. Ricardo de Sousa ${ }^{1,2}$ and N. S. Branco ${ }^{3}$ \\ ${ }^{1}$ Departamento de Física, Universidade Federal do Amazonas \\ 3000, Japiim, 69077-000, Manaus-AM, Brazil \\ ${ }^{2}$ Departamento de Física, ICEx, Universidade Federal de Minas Gerais \\ Av. Antônio Carlos 6627, CP 702, 30123-970, Belo Horizonte-MG, Brazil \\ 3 Departamento de Física, Universidade Federal de \\ Santa Catarina, 88040-900, Florianópolis-SC, Brazil
}

\begin{abstract}
The phase diagram of the spin-1 Ising model in the presence of a biaxial crystalfield anisotropy is studied within the framework of a variational approach, based on the Bogolyubov inequality for the free energy. We have investigated the effects of a transverse crystal-field $D_{y}$ on the phase diagram in the $T-D_{x}$ plane. Results obtained by using effective-field theory (EFT) on the honeycomb $(z=3)$, square $(z=4)$ and simple cubic $(z=6)$ lattices $(z$ is the coordination number) show only continuous phase transitions, while the variational approach presents first-order and continuous phase transitions for $D_{y}=0$. We have also used the EFT for larger values of $z$ and we observe the presence of tricritical points in the phase diagrams, for $z \geq 7$, in accordance with the variational approach results.
\end{abstract}

Pacs number: 05.70.-a, 64.60.-i, 75.10.Nr; 75.50.Lk

In the past few decades there has been an increasing number of studies on the phase transition of the spin-1 Ising model with a longitudinal crystal-field $1,2,3,4,4,5,6.7$, which is described by the following Hamiltonian:

$$
\mathcal{H}=-J \sum_{<i, j>} S_{i}^{z} S_{j}^{z}+D \sum_{i}\left(S_{i}^{z}\right)^{2}
$$

where the first sum is over pairs of nearest-neighbor spins, the second sum is over all spins on the lattice, $J$ is the exchange interaction, $D$ represents the longitudinal crystal-field, and $S_{i}^{z}$ is the $z$-component of a spin- 1 operator at site $i$. The previous Hamiltonian defines the

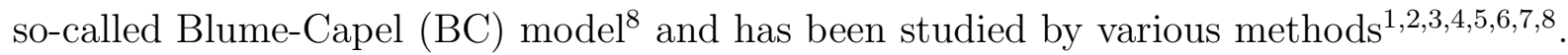


It is well known that when the uniaxial anisotropy, $D$, is greater than $D_{c}=z J / 2(z$ is the coordination number), the system is always in the paramagnetic $(\mathbf{P})$ phase, no matter the temperature. For $D<D_{c}$, there is a ferromagnetic $(\mathbf{F})$ state at low enough temperatures. At $D=D_{c}$, we have a first-order phase transition between the $\mathbf{F}$ and $\mathbf{P}$ phases. For null anisotropy $(D=0)$, the phase diagram in the $(T-D)$ plane, in two and threedimensional lattices, presents a continuous phase transition. As the anisotropy parameter $D$ increases, the critical temperature of this transition decreases. At low temperatures, the system presents a first-order phase transition, with a tricritical point (TCP) separating these two regimes.

For infinite dimension $(d \rightarrow \infty)$, the classical model, Eq. (1), can be exactly solvable, replacing $J$ by $J / N$ ( $N$ is the total number of sites) and the first sum running now over all pairs. This model with long-range interaction is equivalent to using mean-field approximation (MFA) or Curie-Weiss theory. The phase diagram obtained by MFA is qualitatively equivalent to the rigorous results of Monte Carlo simulation ${ }^{\underline{7}}$ in a three-dimensional lattice. The limit $d \rightarrow \infty$ corresponds to the MFA solution and, therefore, all different methods used to treat the $\mathrm{BC}$ model will tend to the MFA results when the coordination number $z$ is increased.

On the other hand, some studies have dealt with the effect of a transverse crystalfield $1,10,11,12,13$. In particular, the presence of a transverse crystal-field in the BC model may change the nature of the phase transition, due to quantum effects. It is known that the determination of thermodynamic properties of quantum models is a non-trivial task, mainly due to the non-commutativity of the operators in the Hamiltonian. Therefore, the use of aproximate methods in these classes of models is always relevant. Moreover, mean-field-like procedures are known to be a good first approximation in describing critical phenomena in three-dimensional models. We then apply a variational approach to study the Hamiltonian:

$$
\mathcal{H}=-J \sum_{<i, j>} S_{i}^{z} S_{j}^{z}+D \sum_{i}\left(S_{i}^{z}\right)^{2}+D^{\prime} \sum_{i}\left(S_{i}^{x}\right)^{2}
$$

where $D^{\prime}$ is the transverse crystal-field anisotropy. Using the spin identity $\left(S_{i}^{x}\right)^{2}+\left(S_{i}^{y}\right)^{2}+$ $\left(S_{i}^{z}\right)^{2}=S(S+1)$, one can rewrite Eq. (2) as

$$
\mathcal{H}=-J \sum_{<i, j>} S_{i}^{z} S_{j}^{z}-D_{x} \sum_{i}\left(S_{i}^{x}\right)^{2}-D_{y} \sum_{i}\left(S_{i}^{y}\right)^{2}
$$


where $D_{x}=D-D^{\prime}, D_{y}=D$ and an irrelevant constant term was dropped out. In particular, for $D^{\prime}=0$ or $D_{x}=D_{y}$ the quantum Hamiltonian, Eq. (3) , reduces to the purely classical model, Eq. (11).

From the experimental point of view, the anisotropy defined in Eq. (3) was shown to play an important role when the thickness of the nonmagnetic TaN interlayer in Fe$\mathrm{TaN} / \mathrm{TaN} / \mathrm{FeTaN}$ sandwiches $\underline{\underline{14}}$ is changed.

On the other hand, theoretical studies of the Hamiltonian described by Eq. (3)), using effective-field theory (EFT) $)^{9,10,11,12,13}$ on honeycomb $(z=3)$, square $(z=4)$ and simple cubic $(z=6)$ lattices, have shown that the system presents only continuous transitions, such that the critical temperature approaches zero at two symmetrical transverse crystalfields, $D_{1 x}=-D_{2 x}$, when $D_{y} \leq 0$. It was observed that, increasing $D_{x}$ from negative values, $T_{c}$ increases from $T_{c}=0$ at $D_{x}=D_{1 x}$, passes through a maximum at $D_{x}<0$ and vanishes again at a positive value of $D_{x}=D_{2 x}$. Another result observed in studies of Hamiltonian (3) is the presence of a TCP for $D_{y}>0$. An important and interesting question is to know if, for $D_{y}=0$, model (3) has a TCP in the phase diagram in the $T-D_{x}$ plane, for large values of $z$.

So, we study here the model described by Eq.(3), which we treat by employing the variational approach based on the Bogolyubov inequality for the free energy. The Bogolyubov variational principle for the free energy is given by

$$
\mathcal{F} \leq \mathcal{F}_{o}+\left\langle\mathcal{H}-\mathcal{H}_{o}(\eta)\right\rangle_{o} \equiv \Phi(\eta)
$$

where $\mathcal{H}$ is the Hamiltonian of the model we want to treat, $\mathcal{H}_{o}$ is the trial Hamiltonian, which can be exactly solved and depends on the variational parameter $\eta, \mathcal{F}_{o}$ is the free energy associated with $\mathcal{H}_{o}$, and $\langle\cdots\rangle_{o}$ is the thermal average over the ensemble defined by $\mathcal{H}_{o}$. The approximate free energy is given by the minimum of $\Phi(\eta)$ with respect to $\eta$.

We use a trial Hamiltonian which includes clusters of one spin, namely:

$$
\mathcal{H}_{o}(\eta)=-\eta \sum_{i} S_{i}^{z}-D_{x} \sum_{i}\left(S_{i}^{x}\right)^{2}-D_{y} \sum_{i}\left(S_{i}^{y}\right)^{2}
$$

Using Eqs. (3) and (5) into Eq. (44), we obtain the variational free energy per spin:

$$
\bar{\Phi}(\eta) \equiv \frac{\Phi(\eta)}{N}=\eta m-z J \frac{m^{2}}{2}-k_{B} T \ln Z_{o}(\eta)
$$


with the partition function

$$
\begin{aligned}
Z_{o} & =\operatorname{Tr}\left\{e^{\beta\left[\eta S_{i}^{z}+D_{x}\left(S_{i}^{x}\right)^{2}+D_{y}\left(S_{i}^{y}\right)^{2}\right]}\right\} \\
& =\exp (2 \beta D)+2 \exp (\beta D) \cosh \left(\beta \sqrt{\Delta^{2}+\eta^{2}}\right)
\end{aligned}
$$

where $D=\left(D_{x}+D_{y}\right) / 2, \Delta=\left(D_{x}-D_{y}\right) / 2$, and $m=\left\langle\frac{1}{N} \sum_{i} S_{i}^{z}\right\rangle_{o}$ is the magnetization per spin.

Minimizing Eq. (6) , we obtain the variational parameter $\eta=z J m$, and the Landau free energy is given by:

$$
\begin{aligned}
\Psi(m) \equiv-\beta \bar{\Phi}(\eta) & \\
& =\quad-z K \frac{m^{2}}{2}+\ln [\exp (2 \beta D)+2 \exp (\beta D) \cosh (W)]
\end{aligned}
$$

with the equation of state (magnetization):

$$
m=\left(\frac{z K m}{W}\right) \frac{2 \sinh (W)}{\exp (\beta D)+2 \cosh (W)},
$$

where $K=\beta J$ and $W=\sqrt{(\beta \Delta)^{2}+(z K m)^{2}}$.

To analyze the second-order phase transition and the TCP, we expand the free energy in powers of $m$ :

$$
\Psi(m) \simeq a_{2} m^{2}+a_{4} m^{4}+a_{6} m^{6}+\cdots
$$

where the coefficients $a_{p}=\frac{1}{p !}\left(\frac{\partial^{p} \Psi}{\partial m^{p}}\right)_{m=0}$ are functions of the parameters $D_{x}, D_{y}$ and $K$.

The continuous phase transition occurs with the breakdown of the order parameter $m$ (i. e., $m \rightarrow 0$ ). So the phase transition boundaries of the $\mathbf{F}$ phase are determined from the zero point of the coefficient of the second-order term in Eq. (10), i.e., $a_{2}=0$ and $a_{4}>0$. To obtain the phase diagram using MFA in all interval of the parameters $D_{x}$ and $D_{y}$, first we compute the free energies, Eq. (8) , for the $\mathbf{F}(m \neq 0)$ and $\mathbf{P}(m=0)$ phases. All the results presented in this section were obtained from numerical solutions of Eqs. (8) and (9). First-order transitions correspond then to the locus on the phase diagram where free energies are equal (i.e., $\left.\Psi_{F}(m)=\Psi_{P}(0)\right)$, with $m=m_{c} \neq 0$, while for continuous phase transitions, $m_{c}=0$. The TCP - in which the phase transition changes from continuous to first-order - is determined by $a_{2}=a_{4}=0$ and $a_{6}>0$.

Defining the dimensionless parameters $\tau \equiv k_{B} T / z J, \delta_{x}=D_{x} / z J$, and $\delta_{y}=D_{y} / z J$, the numerical results for the phase diagram in the $\tau-\delta_{x}$ plane, for different values of $\delta_{y}$, are 
shown in Fig. 1. We chose to depict the values $\delta_{y}=0.5$ (curve (a)), 0.0 (curve (b)) and -0.50 (curve (c)). In this figure, the solid and traced lines correspond to continuous and first-order phase transitions respectively. The filled dots are the TCP. Within the studied region of transverse crystal-field $\delta_{x}$, we could not find any point satisfying the TCP condition $\left(a_{2}=a_{4}=0\right)$ for the model with $\delta_{y}<0$. The situation is different for $\delta_{y} \geq 0$ : when we increase $\delta_{x}$ from negative values the transition temperature $\tau_{c}$ increases, passes through a maximum and satisfies the TCP condition at the points $\left(\delta_{x T}, \tau_{T}\right)=(0.9472,0.2845)$ and $(0.4243,0.3330)$ for $\delta_{y}=0$ and 0.50 , respectively. Thus, we may conclude that the Ising model with a transverse crystal-field exhibits a TCP on the $\tau$ versus $\delta_{x}$ curve, when the transverse crystal field $\delta_{y}$ is null. This is in contrast to EFT results for coordination numbers $z=3,4$ and $6 \underline{9,10,11}, \underline{12}, 13$, for which no TCP is found for any value of $\delta_{y}$.

Some authors 12,13 have used the condition $a_{2}=0$ and $a_{4}<0$ to determinate the firstorder line, but this condition corresponds to the stability line of the $\mathbf{P}$ phase. One can note that it is not possible to calculate first-order lines based on the equation of state (91) alone, because in this case one has $m \neq 0$ at the transition point. Therefore, the first-order line is not correctly obtained using the EFT $-10,11,12,13$ : only critical lines and TCP can be obtained with this procedure, since an expression for the free energy is not available. In order to illustrate the difference between the two procedures, when it comes to locate first-order lines, we show in the inset of Fig. 1 the phase diagram for $\delta_{y}=0$, using the free energy method (traced line, method I) and the condition $a_{2}=0$ and $a_{4}<0$ (dotted line, method II). We note, for example, that at zero temperature $(\tau=0), \delta_{x c}=1.0$ and $\delta_{x c}=2.0$ from methods I and II, respectively.

To access the influence of quantum effects, we can compare our results for $\delta_{y}=0$ with the value of the TCP for the Blume-Capel model using MFA, which is equivalent to replacing $\delta_{x}=\delta_{y}=\delta$ in Eqs. (91) and (10); for the latter, the coordinates of the TCP are $\left(\delta_{T}=0.462\right.$, $\left.\tau_{T}=0.333\right)^{\stackrel{8}{*}}$. This result for the TCP in the Blume-Capel model is different from the one on the Ising model with a transverse crystal-field and $\delta_{y}=0$, namely $\left(\delta_{x T}=0.9472, \tau_{T}=\right.$ 0.2845). In order to illustrate the difference between the two models, in Fig. 2 we show the phase diagram in the $\tau-\delta$ plane for the longitudinal (curve (b), where $\delta_{x}=\delta_{y}=\delta$ ) and transverse (curve (a), where $\delta_{y}=0$ and $\delta_{x}=\delta$ ) crystal-field cases. Particularly, the result for the transition temperature at $\delta=0$ is $\tau_{c}=2 / 3$, and for $\tau=0$ (ground state), we obtain $\delta_{c}=1 / 2$ and 2 for the transverse (a) and longitudinal (b) Blume-Capel models, respectively. 
FIG. 1: Phase diagram in the $\tau-\delta_{x}$ plane for the spin-1 Ising model with a biaxial crystal-field using MFA, for the following values of $\delta_{y}$ : 0.50 (a), 0.0 (b) and -0.5 (c). The continuous and traced lines correspond to continuous and first-order phase transitions, respectively. Filled dots represent tricritical points. The inset shows a comparison of the first-order line obtained by using the free energy (traced line) and condition $a_{2}=0$ and $a_{4}<0$, which corresponds to an unstable solution for $\delta_{y}=0$ (dotted line).

For negative values of $\delta$, the transition line for the Blume-Capel model extends to $\delta \rightarrow-\infty$ and its critical temperature is always finite. The system is in the ordered (ferromagnetic) phase for $\tau<\tau_{c}(\delta)$ while it is in a disordered (paramagnetic) phase for $\tau>\tau_{c}(\delta)$. On the other hand, when the transverse crystal-field anisotropy $\delta \rightarrow-\infty$, we have a paramagnetic state in all temperatures. In the case of a transverse crystal-field, the critical temperature goes to zero at the quantum critical point $\delta_{c}=-2.0$, which is symmetrical to the first-order phase transition point at positive $\delta, \tau=0, \delta_{c}^{\prime}=2.0$.

We have extended the EFT approach on model (3), to investigate the possible existence 
FIG. 2: Phase diagram in the $\tau-\delta$ plane for the spin-1 Ising model with longitudinal (a) and transverse (b) crystal-field using MFA. The continuous and traced lines correspond to continuous and first-order phase transitions, respectively. The black points represents the TCP.

of a TCP for some values of the coordination number. We note the absence of this point in the region $\delta_{y} \leq 0$ and for values of $z \leq 6$, in accordance with previous results $9,10,11,12,13$. However, we verify the presence of a TCP for lattices with $z \geq 7$. In order to illustrate the presence of this TCP, we analyze the bcc $(z=8)$ and fcc $(z=12)$ lattices and, by using the condition $a_{2}=a_{4}=0$ and $a_{6}>0$, we obtain the coordinates of the TCP: $\left(\delta_{x T}, \tau_{T}\right)=(1.0817,0.1708)$ and $(1.0111,0.2177)$, respectively. We compare the results of the TCP obtained by EFT with the MFA values $(0.9472,0.2845)$ and we verify that, with the increase of the coordination number, the TCP tends to the MFA values, as expected.

In summary, we present mean-field calculations based on the Bogolyubov inequality for the free energy, to obtain the phase diagram of the spin-1 Ising model with biaxial crystalfield anisotropy. We verify that for zero transverse crystal-field $\delta_{y}$, the system exhibit a 
TCP in the phase diagram for all values of $z$, while the results obtained by effective field theory ${ }^{9}, 10,11,12,13$ for coordination number $z=3,4$ and 6 present only continuous phase transitions. We generalize the EFT for lattices with large values of $z$, and it was observed the presence of a TCP for $z \geq 7$. With the increase of $z$, the EFT results tends to the MFA solution. In order to investigate the MFA results by using the differential operator technique, we suggest the use of the correlated-EFT ${ }^{17}$ or the EFT in a finite cluster with two spins $\frac{18}{}$. Finally, owing to the mathematical simplicity of our formulation, we hope that the present work will be potentially useful for studying more complicated systems, including, for example, a transverse magnetic field. These generalizations are under investigation.

ACKNOWLEDGMENTS: The authors acknowledge valuable discussions with Dr. J. A. Plascak. This work was partially supported by CNPq, FAPEAM and FAPESC (Brazilian agencies).

1 A. F. Siqueira and I. P. Fittipaldi, Physica A 138, 592 (1986).

2 Y. Yamada, N. Todoroki, and S. Miyashita, Phys. Rev. B 69, 024103 (2004).

3 N. S. Branco, Phys. Rev. B 60, 1033 (1999).

4 J. W. Tucker, J. Mag. Mag. Mater 183, 299 (1998).

5 T. Kaneyoshi, Phys. Rev. B 34, 7866 (1986); ibid J. Phys. C 19, L557 (1986).

6 H. P. Dong and S. L. Yan, J. Mag. Mag. Mater. 308, 90 (2007), and references therein.

7 J. A. Plascak, D. P. Lara, S. J. Ferreira, and O. F. A. Bonfim, J. Mag. Mag. Mater. 177, 163 (1998).

8 M. Blume, Phys. Rev. 141, 517 (1966); H. W. Capel, Phyisica 32, 966 (1966).

9 J. Oitmaa and A. M. A. V. Brasch, Phys. Rev. B 67, 172402 (2003).

10 Q. Zhang, G. Z. Wei, and Y. Q. Liang, J. Mag. Mag. Mater. 253, 45 (2002).

11 W. Jiang, G. Z. Wei, and Q. Zhang, Physica A 329, 161 (2003).

12 L. Xu and S. L. Yan, Solid State Comm. 142, 159 (2007).

13 J. Wei, W. Xi-Kum, and Z. Qiang, Chin. Phys. 15, 1009 (2006).

14 H. B. Nie, C. K. Ong, J. P. Wang, and Z. W. Li, J. Appl. Phys. 93, 7252 (2003).

15 Subir Sachdev, Quantum Phase Transitions (Cambridge University Press, Cambridge, 2001). 
16 M. A. Continentino, Quantum Scaling in Many Body Systems (World Scientific, Singapore, 2001).

17 T. Kaneyoshi, Physica A 164, 730 (1990).

18 M. Jurcisin, A. Bobak, and M. Jascur, Physica A 224, 684 (1996). 


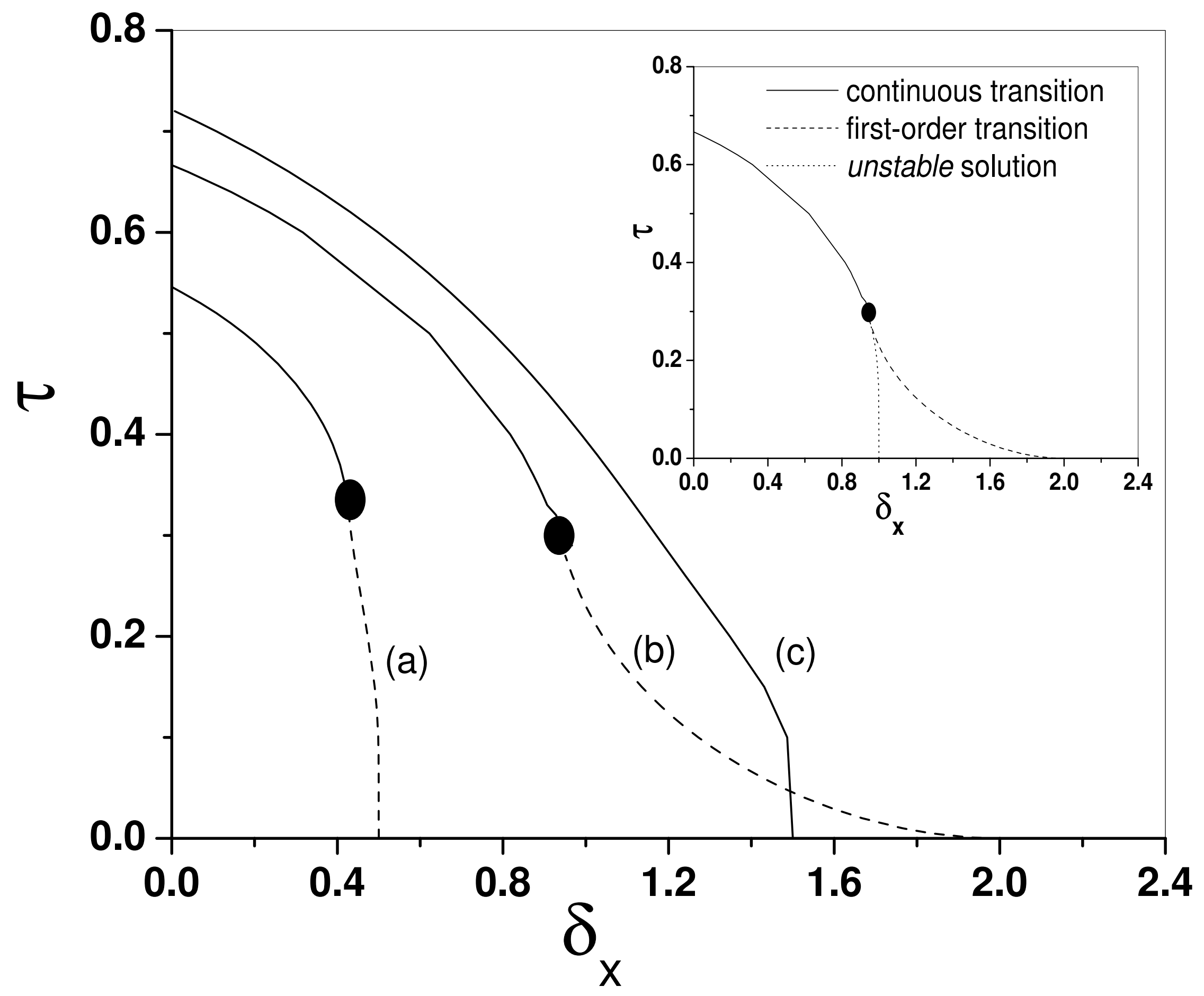




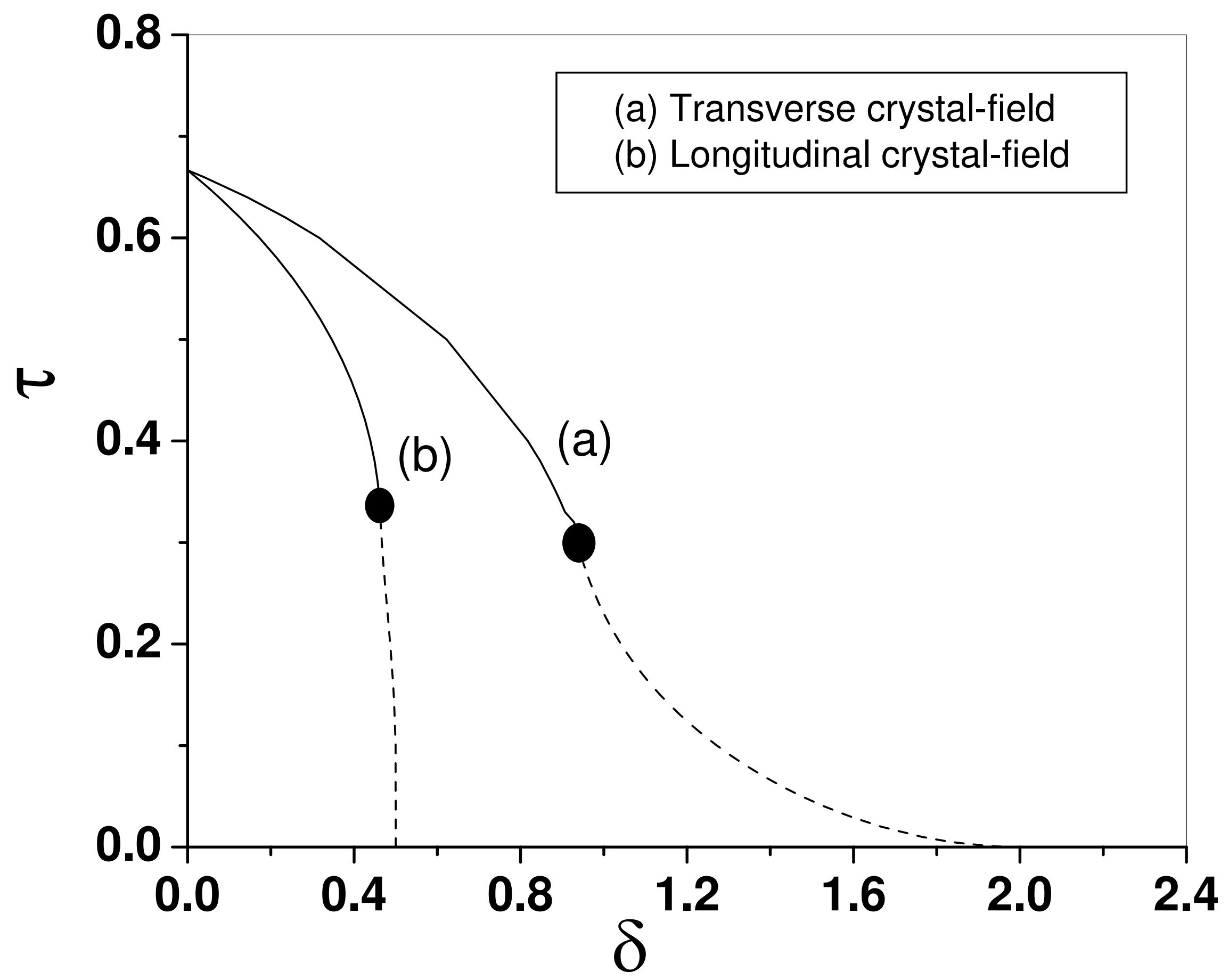

